\title{
The role of SI00A9 in distinguishing malignant melanoma from dysplastic and common nevi
}

\begin{abstract}
Background: Increased S100A9 expression has been found at the deep invasive edge of melanomas, as well as in the upper layers of the epidermis above and bordering melanomas. However, no studies to date have examined the potential clinical utility of using S100A9 to differentiate between nevi and melanoma. The aim of this study is to examine S100A9 IHC expression in common nevi, dysplastic nevi, and melanomas in order to determine whether the S100A9 marker could help distinguish these lesions.

Methods: 13 primary cutaneous melanomas and 19 nevi were retrieved from the archives of Loyola University Medical Center. S100A9 IHC expression was classified according to three patterns: diffuse, linear, and irregular.

Results: $53 \%$ of malignant melanoma samples stained in a diffuse pattern, the majority of dysplastic nevi stained in a linear pattern $(60 \%)$, while benign nevi stained primarily in an irregular pattern $(50 \%)$. All diffusely staining melanoma lesions exhibited a Below depth $>1 \mathrm{~mm}$, unlike linear and irregularly staining melanomas.

Conclusion: The diffuse presence of S100A9 stain and increased depth of invasion in melanoma lesions may support a "concentration effect" hypothesis of S100A9 function. Our results also indicate that diffuse staining with S100A9 has a specificity of $73.7 \%$ and sensitivity of $53.8 \%$ for detection of melanoma.
\end{abstract}

Keywords: malignant melanoma, immunohistochemistry, common nevi, dysplastic nevi, S100A9 stain
Volume I Issue 2 - 2017

\author{
Solomiya Grushchak, Arielle R Gray, Cara \\ Joyce, Kelli A Hutchens and Kumaran \\ Mudaliar
}

Stritch School of Medicine, Loyola University Chicago, USA

Correspondence: Solomiya Grushchak, MD Candidate Class of 2018, Loyola University Chicago, 2160 S I st Ave, Maywood, IL 60 153, Tel 847-975-5594, Email sgrushchak@luc.edu

Received: May 30, 2017 | Published: June 29, 2017

\section{Introduction}

A high degree of homology exists between the highly conserved S100 protein family, and researchers continue to investigate the precise function and structure of each gene. The S100 proteins are small acidic proteins $10-12 \mathrm{kDA}$ in size that consist of two helixloop-helix Calcium binding domains called EF-hands. ${ }^{1}$ Although they exhibit a high degree of homology, S100 proteins are distinguished by the specific amino acids that compose their hinge region and C-terminal extension ${ }^{1}$. These two regions are responsible for forming the target protein complex, which determines the precise function of each S100 gene. ${ }^{2}$ S100B, expressed primarily by melanocytes and Langerhans cells, is routinely used in practice to highlight melanocytic lesions and melanoma metastases in the clinical setting. ${ }^{3}$ However, the S100B diagnostic kits have been found to cross-react with other S100 proteins such as S100A5 and S100A6. Researchers have speculated whether other S100 proteins could be used as sensitive and specific markers to distinguish nevi from malignant melanoma.

The S100A9 protein, Calprotectin, is a calcium binding protein within the S100 family of genes that forms heterodimer with S100A8 within cells of myelomonocytic origin. ${ }^{1}$ S100A9 is constitutively expressed in myeloid progenitor cells such as neutrophils, dendritic cells, monocytes, and platelets as well as mucosal epithelium and skin. ${ }^{4}$ Increased expression of S100A9has been found in cutaneous squamous cell carcinoma and in tumors of the breast, lung, stomach, colon, pancreas, and prostate. Hibino et al..$^{5}$ studied the positive upregulation of S100A9 in the invasive edge and adjacent epidermis of malignant melanoma. Their data suggested that S100A9 was strongly associated with cell surface glycoprotein, EMMPIRIN. S100A9 bound to EMMIRIN was found to induce the production of several matrix metalloproteinase responsible for degrading the basement membrane and enhancing the metastatic ability of malignant melanoma at the invasive edge. Pro-inflammatory factors such as VEGF-A, TGF-B, and TNF-A induce selective expression of S100A9, which then directs the migration of malignant cells to pre-metastatic locations. ${ }^{6}$

More recent in vitro and in vivo studies of S100A9have led to the hypothesis that the regulation of tumorigenesis may be largely dependent on specific S100A9 concentration. ${ }^{7}$ In studies evaluating serum levels of S100A9 in mice with chemically-induced squamous cell carcinoma, tumor bearing mice had lower levels of S100A9 in serum than those seen in healthy controls. ${ }^{8}$ In contrast, multiple human studies have found that S100A9 is fact constitutively expressed at high levels in human squamous cell carcinomas. ${ }^{9}$ The tumor effects of S100A9, whether anti-tumor or pro-tumor, are perhaps concentration dependent or "model-dependent" (human vs. murine). ${ }^{10}$

In this study, we focus on the up-regulation of S100A9 in the epidermis of melanomas. Although previous studies show increased expression of this protein at the invasive edge and the epidermis of melanomas, epidermal staining with S100A9 has not been evaluated for its histologic utility indistinguishingnevi from malignant melanoma. S100A9 epidermal staining patterns are evaluated in common nevi, dysplastic nevi, and malignant melanoma to assess the potential discriminatory value in helping to distinguish these lesions. 


\section{Methods}

\section{Sample preparation}

Following institutional review board-authorization, 13 primary cutaneous melanomas and 19 nevi were retrieved ( 5 common nevi, 5 mild dysplastic, 4 moderate dysplastic, and 5 severe dysplastic), (Figures 1-3). 5- $\mu \mathrm{m}$ formalin-fixed, paraffin-embedded sections were cut and mounted onto positively charged slides. Single IHC S100A9 staining was performed using the Leica Bond III protocol. All antibodies were purchased from Leica Biosystems (Buffalo Grove, IL). A board certified dermatopathologist evaluated S100A9 staining of melanocytes and keratinocytes in the epidermis of each sample. The pattern of staining was classified according to one of three categories: diffuse epidermal staining Figure 4, linear basement membrane staining Figure 5, and irregular patchy epidermal staining Figure 6.

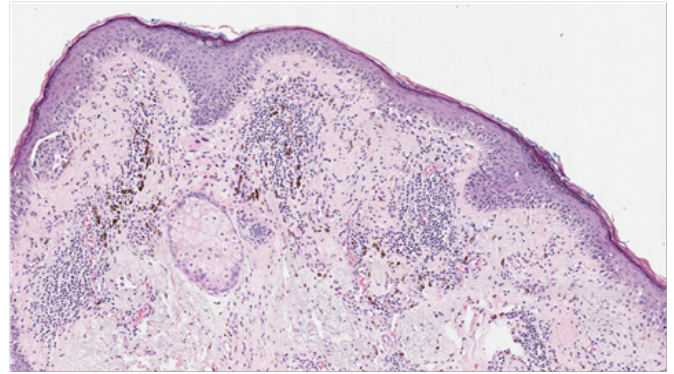

Figure I H\&E stain of malignant melanoma in situ of lesion A (hematoxylin and eosin, 40x).

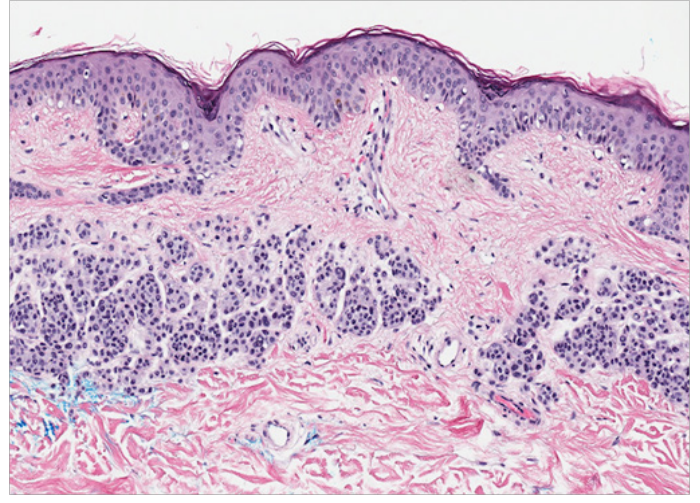

Figure 2 H\&E stain of mildly dysplastic nevi of lesion B(hematoxylin and eosin, 40x).

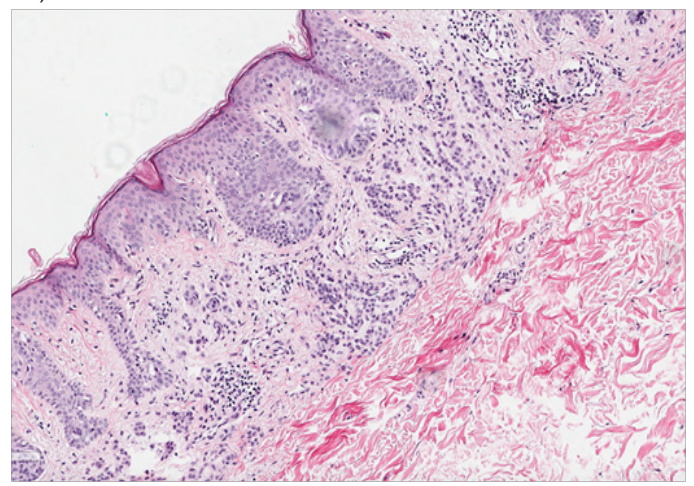

Figure $3 \mathrm{H} \& \mathrm{E}$ stain of compound nevi of lesion C(hematoxylin and eosin, $40 \mathrm{x})$.

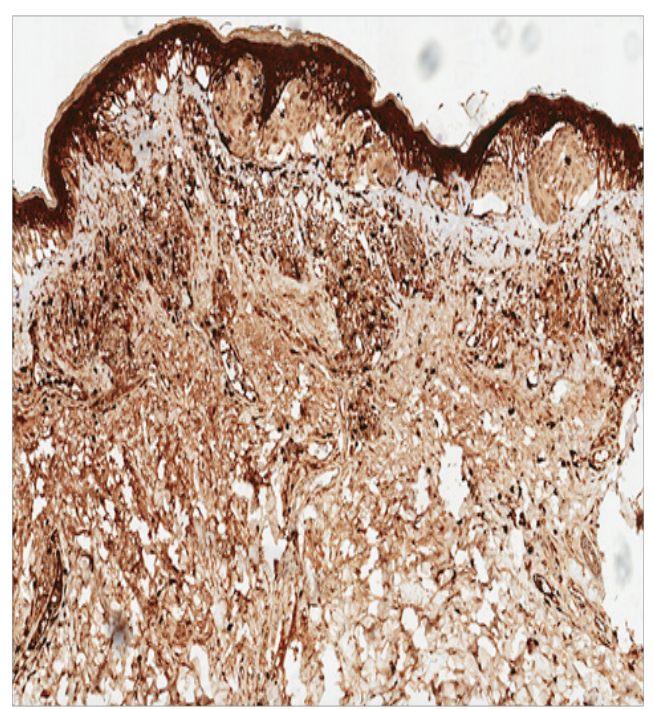

Figure 4 Diffuse epidermal SI00A9 stain of malignant melanoma in situ of lesion $\mathrm{A}$.

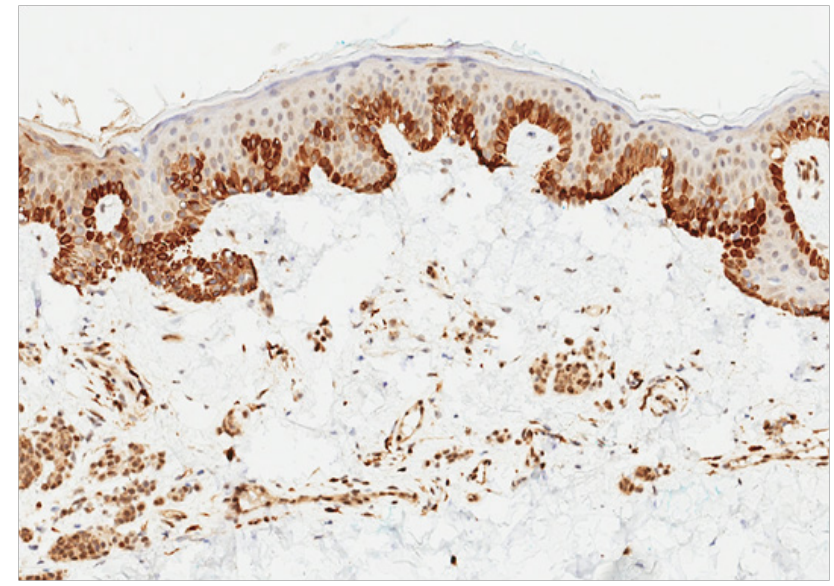

Figure 5 Linear SI00A9 stain of mildly dysplastic nevi of lesion B.

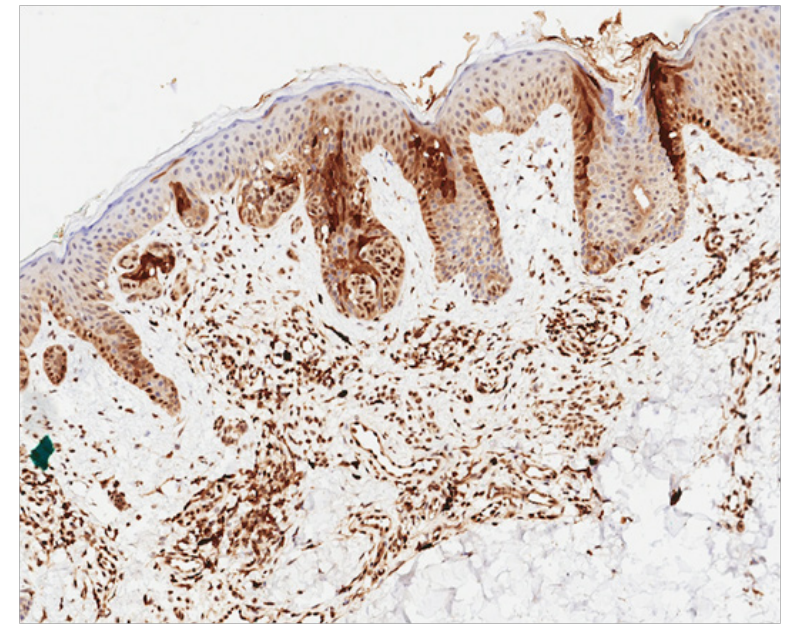

Figure 6 Irregular S100A9 stain of compound nevi of lesion C. 


\section{Statistical analysis}

Clinical characteristics were presented as means and standard deviations or counts and percentages. Statistical significance of differences by sample type were assessed via a t-test (age), a chisquare test (sex), and Fisher's exact tests (all others). Diffuse patterns were compared to combined linear and irregular patterns using Fisher's exact test for site, type, and solar elastosis and with an exact Wilcoxon rank sum test for Breslow's depth. Statistical analyses were performed using SAS 9.4 (Cary, NC).

\section{Results}

In all cases, nevus cells and melanoma cells were positive for S100A9. $53.8 \%$ of the melanoma samples demonstrated a diffuse epidermal pattern (7/13), 15.4\% showed linear epidermal staining $(2 / 13)$, and $30.8 \%$ showed irregular patchy staining (4/13) (Table 1). Of the compound melanocytic nevi, $20 \%$ stained in a diffuse epidermal pattern (1/5), 40\% stained in a linear pattern $(2 / 5)$, and $40 \%$ stained in an irregular pattern (2/5) (Table 1$)$. Of the mildly dysplastic compound nevi, $20 \%$ stained in a diffuse epidermal pattern $(1 / 5)$, $20 \%$ stained in a linear pattern (1/5), and $60 \%$ stained in an irregular pattern (3/5) (Table 1). Of the moderately dysplastic compound nevi, $50 \%$ stained in a diffuse epidermal pattern $(2 / 5), 0 \%$ stained in a linear pattern $(0 / 5)$, and $50 \%$ stained in an irregular pattern (2/5) (Table 1$)$. When grouping together benign nevi not worrisome for melanoma (compound, mildly dysplastic, and moderately dysplastic), $28.5 \%$ stained in a diffuse pattern (4/14), $21.4 \%$ stained in a linear pattern $(3 / 14)$, and $50 \%$ stained in an irregular pattern (7/14). Of the severely dysplastic compound nevi, $20 \%$ stained in a diffuse epidermal pattern $(1 / 5), 60 \%$ stained in a linear pattern (3/5), and $20 \%$ stained in an irregular pattern $(1 / 5)$.

There was no evidence of a significant difference in staining patterns among the melanoma and nevi $(\mathrm{p}=0.35)$. When comparing the melanomas and nevi, melanoma lesions were from older patients (61.1 vs. 38.2 years old, $\mathrm{p}<0.001)$, less likely to be taken from the trunk $(46.2 \%$ vs. $89.5 \%, p=0.008)$, and more likely to have solar elastosis $(61.5 \%$ vs. $10.5 \%, p=0.005)$ (Table 2). Comparing diffuse to linear and irregular staining patterns of melanomas, diffuse patterns were more often observed on the trunk $(71.4 \%$ vs. $16.7 \%, \mathrm{p}=0.012)$ and had a greater median below depth $(1.58 \mathrm{~mm}$ vs. $0.31 \mathrm{~mm}, \mathrm{p}=0.005)$ (Table 3). A diffuse S100A9 epidermal staining demonstrated sensitivity of $53.8 \%$ and $73.7 \%$ specificity in the detection of melanomas.

Table I Staining patterns of nevi and melanoma lesions

\begin{tabular}{lllll}
\hline & Staining pattern & & & \\
\hline Diagnosis & Diffuse, n (\%) & Linear, n (\%) & Irregular, n (\%) & Total, n \\
\hline Compound Melanocytic Nevi & $1(20.0)$ & $2(40.0)$ & $2(40.0)$ & 5 \\
Mildly Dysplastic Compound Nevi & $1(20.0)$ & $1(20.0)$ & $3(60.0)$ & 5 \\
Moderately Dysplastic Compound Nevi & $2(50.0)$ & $0(00.0)$ & $2(50.0)$ & 4 \\
Severley Dysplastic Compound Nevi & $1(20.0)$ & $3(60.0)$ & $1(20.0)$ & 5 \\
Malignant Melanoma & $7(53.8)$ & $2(15.3)$ & $4(30.7)$ & 13 \\
\hline
\end{tabular}

Table 2 Clinical characteristics of melanoma and nevi samples

\begin{tabular}{llll}
\hline & Melanoma $\mathbf{n = 1 3}$ & Nevi n=19 & p-value \\
\hline Age, mean(SD) & $61.1(16.4)$ & $38.2(16.6)$ & $<0.001$ \\
Female, n (\%) & $6(46.2)$ & $10 .(54.3)$ & 0.72 \\
Location, n(\%) & & \\
Head/neck & $2(15.4)$ & $0 .(0.0)$ & 0.008 \\
Trunk & $6(46.2)$ & $17(89.5)$ & \\
Lower Extremity & $2(15.4)$ & $2(10.5)$ & \\
Upper Extremity & $3(23.1)$ & $0(0.0)$ & \\
Solar elastosis, $\mathrm{n}(\%)$ & $8(61.5)$ & $2(10.5)$ & 0.0005 \\
Staining pattern, $\mathrm{n}(\%)$ & & & \\
Diffuse & $7(53.8)$ & $5(26.3)$ & 0.35 \\
Linear & $2(15.4)$ & $6(31.6)$ & \\
Irregular & $4(30.8)$ & $8(42.1)$ & \\
\hline
\end{tabular}


Table 3 Clinical characteristics of melanoma by staining pattern

\begin{tabular}{llll}
\hline Staining pattern & Diffuse $\mathbf{n = 7}$ & Linear/Irregular n=6 & p-value \\
\hline Location, n(\%) & & & 0.012 \\
Head/Neck & $2(28.6)$ & $0(0.0)$ & $1(16.7)$ \\
Trunk & & $5(71.4)$ & $2(33.3)$ \\
Lower Extremity & $5(71.4)$ & $0(0.0)$ & $3(50.0)$ \\
Upper Extremity & $0(0.0)$ & $0(0.0)$ & $4(66.7)$ \\
Solar elastosis, n(\%) & $0(0.0)$ & $4(57.1)$ & $0.31(0.27, .40)$ \\
Depth (mm), Median (range) & $4(57.1)$ & $1.58(1.00,5.00)$ & 0.005 \\
Type of melanoma, n (\%) & $1.58(1.00,5.00)$ & & $4(66.7)$ \\
Malignant/superficial & & & $0(0.0)$ \\
Spreading & $5(71.4)$ & $5(71.4)$ & \\
In situ & $1(14.3)$ & $1(14.3)$ & $1(14.3)$ \\
Unspecified & $1(14.3)$ & & 0.16 \\
\hline
\end{tabular}

\section{Discussion}

The S100A9 protein, Calprotectin, is a protein that forms heterodimer within cells of myelomonocytic origin. With the exception of some epithelial tissue, S100A9 expression is found at low levels in healthy individuals and rises in response to epidermal injury in acute and chronic inflammation, UV exposure, tape stripping, and other skin stresses. ${ }^{3}$ The homodimer functions as a sensor and regulator of migration, proliferation, and differentiation of inflammatory cells. ${ }^{4}$ Experimental data indicate that a positive feedback mechanism, initiated and regulated by S100A9, is responsible for the inflammatory environment seen in many pathologic conditions (e.g. rheumatoid arthritis, systemic lupus erythematosus, giant cell arteritis, psoriasis, inflammatory bowel disease, and multiple sclerosis). ${ }^{5}$ During inflammation, myeloid cells promote an increase in intracellular calcium, resulting in the translocation of S100A9 to the membrane surface. The expression of S100A9 enhances leukocyte recruitment. ${ }^{5}$ This positive feedback mechanism ensures an aggressive inflammatory environment for successful tumor proliferation and metastases. ${ }^{6}$ In our study, the majority of non-worrisome nevi (compound nevi, mildly dysplastic nevi, moderately dysplastic nevi) stained primarily in an irregular pattern, severely dysplastic nevi stained a linear pattern (Figure 5), and the majority of melanoma lesions stained in a diffuse epidermal pattern. The dysplastic nevi did not appear to show increased levels of diffuse staining with grading of atypia. As severely dysplastic nevi could be histologically confused for melanoma and vice versa, a difference in staining pattern between severely dysplastic nevi and melanoma could be of clinical utility. From our results, a diffuse pattern would be more compatible with melanoma while a linear pattern would be more compatible with a severely dysplastic nevus. Hibino et al. reported diffuse staining of S100A9 in the epidermis overlying the melanoma and in the neighboring the epidermis. Additionally, the pigmented nevi in their study did not show significant S100A9 stain in the epidermis, and was similar to the irregular staining pattern we observed in our samples of benign nevi. Both of these findings correlate well with the results of our study.
We found that the depth of invasion in diffusely staining melanoma lesions was $>1 \mathrm{~mm}$. Diffuse staining with S100A9 is correlated with a deeper invasion of the dermis by malignant cells. Heavy staining with S100A9 stain in melanoma lesions may support the "concentration effect" hypothesis of S100A9 function that higher levels of S100A9 may be correlated with increased tumor burden. ${ }^{7}$ In conclusion, the diffuse staining pattern of S100A9, although specific, is not highly sensitive. Due to our low sample size and lack of statistical significance, future research should investigate whether this marker has utility when diagnosing ambiguous lesions. Stratification into specific staining patterns with a greater number of samples may show that S100A9 has clinical use in both diagnosis and prognosis of malignant melanoma.

\section{Acknowledgements}

The authors thank all individuals who contributed to this study.

\section{Conflict of interest}

The author declares no conflict of interest.

\section{References}

1. Heizmann CW, Fritz G, Schafer B. S100 proteins: Structure, functions and pathology. Front Biosci. 2002;7(1):1356-1368.

2. Donato R. S100: A multigenic family of calcium-modulated proteins of the EF-hand type with intracellular and extracellular functional roles. Int $J$ Biochem Cell Biol. 2001;33(7):637-668.

3. Eckert RL, Broome A, Ruse M, et al. S100 proteins in the epidermis. $J$ Invest Dermatol. 2004;123(1):23-33.

4. Bresnick AR, Weber DJ, Zimmer DB. S100 proteins in cancer. Nature reviews Cancer. 2015;15(2):96-109.

5. Gebhardt C, Németh J, Angel P, et al. S100A8 and S100A9 in inflammation and cancer. Biochem Pharmacol. 2006;72(11):1622-1631.

6. Hibino T, Sakaguchi M, Miyamoto S. S100A9 is a novel ligand 
of EMMPRIN that promotes melanoma metastasis. Cancer Res. 2013;73(1):172-183.

7. Srikrishna G. S100A8 and S100A9: New insights into their roles in malignancy. J Innate Immun. 2012;4(1):31-40.

8. McNeill E, Hogg N. S100A9 has a protective role in inflammation $\square$ induced skin carcinogenesis. International Journal of Cancer. 2014;135(4):798808
9. Maelandsmo GM, Floerenes VA, Mellingsaeter T, et al. Differential expression patterns of S100A2, S100A4, and S100A6 during progression of human malignant melanoma. International journal of cancer. 1997;74(4):464-469.

10. Farmer ER, Gonin R, Hanna MP. Discordance in the histopathologic diagnosis of melanoma and melanocytic nevi between expert pathologists. Hum Pathol. 1996;27(6):528-531. 